\title{
Obstetric and gynaecological factors in susceptibility to peripheral joint osteoarthritis
}

\author{
Alan J Silman, Jason Newman
}

Osteoarthritis represents the final common pathway of a number of different pathological processes. Epidemiological studies for risk factors are therefore liable to produce inconsistent observations. Despite this, there is considerable evidence from cross sectional prevalence studies based on radiographical surveys of whole populations suggesting an increased occurrence in females. Typical findings include a more rapid rise in the occurrence with age in females, particularly of small joint hand osteoarthritis, with plateauing after the age of around 60 years. By contrast, there is a much slower rise in males, which continues into the seventh and eighth decade. ${ }^{1}$ It is for this reason that many have suggested there might be a hormonal basis to the development of osteoarthritis.

The occurrence of joint symptoms around the time of the menopause has been documented by many observers over the past 100 years. Indeed in a quotation attributed to Haygarth in 1805, it was stated, "Nodosities of joints are almost peculiar to women and begin when menses naturally cease". ${ }^{2}$ There have been many clinical series subsequently published of female patients presenting with fairly rapid onset of polyarticular nodal osteoarthritis concurrently with the onset of menopausal symptoms and the cessation of menstruation.

\section{Is there biological evidence for a sex hormone linked susceptibility?}

There have been many studies, particularly of animal models, suggesting that sex hormones can modify cartilage metabolism. Such observations may be relevant to human disease.

Oestrogen receptors have been found in the articular cartilage in a number of species. ${ }^{34}$ Several animal studies have shown that ovariectomy may increase cartilage breakdown. ${ }^{5}$ More direct evidence of the role of oestrogens comes from experiments carried out over the past 20 years. It was originally shown that oestradiol may suppress proteoglycan synthesis in animal models. ${ }^{6}$ In further experiments, supraphysiological doses of oestradiol increase cartilage breakdown which is blocked when tamoxifen, an antioestrogen, is given. ${ }^{78}$ In more recent work, ovariectomised rabbits exposed to high, but not low, doses of oestrogens show changes in the articular cartilage such as thinning and fibrillation, typical of human osteoarthritis. ${ }^{9}$ It therefore seems reasonable to conclude that variation in either oestrogen synthesis or control might affect susceptibility to osteoarthritis in human females.
Endogenous hormone production is clearly altered during both menstruation and pregnancy and it is thus reasonable to investigate whether menstrual and obstetric history influences susceptibility to osteoarthritis.

\section{Menstrual history}

AGE AT MENARCHE

There does not appear to be any difference at the age of menarche between women who have developed and those who have remained free of osteoarthritis. ${ }^{10}$ This is true both for women with a generalised nodal osteoarthritis and those with disease restricted to the large lower limb joints. ${ }^{11}$ In all these studies, the age at menarche was identical between the groups.

AGE AT MENOPAUSE

There does not appear either to be any difference in age at menopause between women who have, and have not, developed osteoarthritis. In a study of hospital cases of disease at many joint sites, the mean age at menopause was 48.5 years in the cases and 49 years in the age matched group of controls. ${ }^{10}$ In the Framingham study of knee osteoarthritis, again there was an identical age between those women who developed osteoarthritis and those who remained free of the disease. ${ }^{12}$ Further case-control studies also showed no difference in the age at menopause in women with small hand joint osteoarthritis either with or without lower limb disease. ${ }^{11}$

A more detailed study of the role of age at menopause showed that there was no association with the development of radiologically apparent distal interphalangeal joint osteoarthritis; by contrast, Heberden's nodes were three times more frequent in women whose age at menopause was above 50 years. ${ }^{13}$ Investigation of the very small subgroup of women who had their menopause before the age of $\mathbf{4 0}$ did not show any difference in their susceptibility to knee osteoarthritis compared to women who had a later menopause. ${ }^{14}$ In one study of hip osteoarthritis, there was a slight but statistically non-significant trend of decreasing association of development of osteoarthritis with increasing age at menopause. ${ }^{15}$ In another more recent study of hip osteoarthritis, there was an apparent risk with increasing age at menopause, but this disappeared after adjusting for potentially confounding variables. ${ }^{16}$

\section{Surgical menopause and osteoarthritis}

It was postulated by Spector et al ${ }^{17}$ that there may be an increased association between surgi- 
cal menopause and the development of osteoarthritis. Two explanations were offered. First, that the surgery may be accompanied by oophorectomy, resulting in major alteration in sex hormone level. The alternative hypothesis put forward was that hysterectomy was frequently undertaken for dysfunctional uterine bleeding. This latter condition was frequently a result of abnormal oestrogen activity and therefore hysterectomy is a marker for abnormality in sex hormone production. This hypothesis was based on the results from a case-control study comparing hospital cases with population controls. ${ }^{17}$ The hospital cases had involvement of osteoarthritis at multiple sites, but the diagnosis was not verified. However, in that group there was an odds ratio of 2.9 (95\% confidence interval 1.8 to 4.7$)$ for having had hysterectomy one year before presentation with disease. In a further investigation, these investigators ${ }^{18}$ undertook a retrospective cohort study, recruiting women who had had a hysterectomy some years earlier and comparing their current joint status with a group who had not had this surgery. The results in this study were conflicting. When the authors restricted their analysis to those with radiologically defined disease, medial joint space narrowing of the knee (as defined by the lowest tertile of joint space narrowing) was associated with a threefold increased risk in the hysterectomised group. There was, however, no evidence of an increased risk of osteoarthritis of the knee using the Kellgren and Lawrence grading, or in the small joints of the hands. When the authors analysed their data in relation to those who had both clinical and radiological evidence of disease, there was a threefold increased risk of first carpometacarpal joint osteoarthritis for women with a prior hysterectomy, but no increase for distal or proximal interphalangeal joint disease. ${ }^{18}$

Further investigations have failed to confirm any affect of surgical menopause on risk. In the Framingham study, ${ }^{12}$ a population based study where women who had developed radiographic knee osteoarthritis were compared with those who had not, there was a small but statistically significant reduction in risk in relation to having had a hysterectomy. Similarly, in another population study from The Netherlands ${ }^{13}$ examining distal interphalangeal joint osteoarthritis there was a marked reduction in the susceptibility to Heberden's nodes in those who had had artificial menopause (odds ratio $0.34,95 \%$ confidence interval 0.13 to 0.89 ) and a non-significant reduction in the risk of radiologically observed distal interphalangeal joint disease (odds ratio $0.73,95 \%$ confidence interval 0.37 to 1.45 ). In a fifth study using hospital cases and general practice controls, odds ratios were around unity for nodal generalised osteoarthritis both with and without large joint knee and hip osteoarthritis. ${ }^{11}$ In a further more recent study, no effect of surgical menopause was noted. ${ }^{19}$ Further, in a recent study of hip osteoarthritis a protective effect of hysterectomy was suggested that was unexplained by other confounding variables. $^{16}$
It thus appears that there is no consistent evidence linking surgical menopause, and the factors leading to it, to increasing susceptibility to osteoarthritis. One problem in interpretation, however, is that the reasons for hysterectomy are not consistent between populations, with US groups in particular having a higher operation rate related in part to social factors; in such women the procedure is not necessarily a marker of hormonal dysregulation.

\section{Parity}

There have been relatively few studies, perhaps surprisingly, on the relation between pregnancy history and the subsequent development of osteoarthritis. In three studies with data, ${ }^{1115}$ there was a non-significant suggestion that women who had ever been pregnant had a modest reduction in their subsequent risk of development of osteoarthritis. None of these studies alone produced clear evidence of a protective effect, but pooling the studies covering arthritis at diverse sites, defined in a number of different ways, does suggest an overall protective effect, even if it is somewhat modest.

\section{Summary}

There is clear evidence that the age period coinciding with the peak age of the menopause is associated with an increased prevalence of osteoarthritis and this fits in with clinical observation of high likelihood of presentation at this age. A number of pieces of biological evidence also support the notion that changes in sex hormone status might influence risk of degenerative disease at peripheral joint sites. There do not appear, however, to be any important epidemiological predictors based on menstrual or obstetric history that might be useful in predicting who these women might be.

1 Van Sasse JLCM, van Romunde LKJ, Cats A, Vandenbroucke JP, Valkenburg HA. Epidemiology of osteoarthritis: Zoetermeer survey. Comparison of radiological osteoarthritis in a Dutch population with that in 10 other populations. Ann Rheum Dis 1989;48:271-80.

2 Hayarth J. A clinical history of diseases. Part 2. A clinical history of the nodosity of joints. London: Cadell and Davies, 1805 .

of the nodosity of joints. London: Cadell and Davies, 1805. tors in adult canine articular cartilage. Arthritis Rheum 1982;25:568-73.

4 Sheridan PJ, Aufdemorte TB, Holt GR, Gates GA. Cartilage of the baboon contains estrogen receptors. Rheumatol Int 1985;5:279-81.

5 Da Silva JAP, Larbre J-P, Seed MP, Cutolo M, Villaggio B, Scott DL, et al. Sex differences in inflammation induced cartilage damage in rodents. The influence of sex steroids. cartilage damage in rodents.

6 Rosner IA, Goldberg VM, Getzy L, Moskowitz RW. Effects of estrogen on cartilage and experimentally induced osteoarthritis. Arthritis Rheum 1979;22:52-8.

7 Rosner IA, Malemud CJ, Goldberg VM, Papay RS, Getzy L, Moskowitz RW. Pathologic and metabolic responses of experimental osteoarthritis to oestradiol and an oestradiol antagonist. Clin Orthop 1982;171:280-6.

8 Rosner IA, Goldberg VM, Moskowitz RW. Estrogens and osteoarthritis. Clin Orthop 1986;213:77-83.

9 Tsai C-L, Liu T-K. Oestradiol-induced knee osteoarthritis in ovariectomized rabbits. Clin Orthop 1993;291:295-302.

10 Spector TD, Roman E, Silman AJ. The pill, parity and Spector TD, Roman E, Silman AJ. The pill, parity
rheumatoid arthritis. Arthritis Rheum 1990;33:782-9.

11 Samanta A, Jones A, Regan M, Wilson S, Doherty M. Is osteoarthritis in women affected by hormonal changes or smoking? Br $\mathcal{F}$ Rheumatol 1993;32:366-70.

12 Hannan MT, Felson DT, Anderson J, Naimark A, Kannel WB. Estrogen use and radiographic osteoarthritis of the knee in women. Arthritis Rheum 1990;33:525-32. 
13 Schouten JSAG, van den Ouweland FA, Valkenburg HA. Natural menopause, oophorectomy, hysterectomy and the risk of osteoarthritis in dip joints. Scand $\varsubsetneqq$ Rheumatol 1992; 21:196-200.

14 Anderson J, Felson DT. Factors associated with osteoarthritis of the knee in the First National Health and Nutrition Examination Survey (NHANES-1). Am $\mathcal{f}$ Epidemiol 1988;128:179-89.

15 Tepper S, Hochberg MC. Factors associated with hip osteoarthritis: data from the First National Health and Nutrition Examination Survey (NHANES-1). Am $\mathcal{f}$ Epidemiol 1993;137:1081-8.

16 Hochberg MC, Powell-Threets, SK, Nevitt MC, Lane NE, Cummings SR, Pressman AR, et al. Reproductive and gynaecologic history and osteoarthritis of the hip in elderly women: data from the study of osteoporotic fractures. Arthritis Rheum 1995;38(suppl):S396.

17 Spector TD, Brown GC, Silman AJ. Increased rates of previous hysterectomy and gynaecological operations in women with osteoarthritis. BMF 1988;297:899-901.

18 Spector TD, Hart DJ, Brown P, Almeyda J, Dacre JE, Doyle DV, et al. Frequency of osteoarthritis in hysterectomized women. $\mathcal{f}$ Rheumatol 1991;18:1877-83.

19 Lethbridge-Cejku M, Hochberg MC, Scott WW, Reichle R, Plato CC, Roy TA, et al. Lack of association of reproductive and gynaecologic factors with radiographic features of osteoarthritis of the knee in postmenopausal women: Data from the Baltimore Longitudinal Study of Aging. Arthritis Rheum 1995;38(suppl):S223.

\title{
Sex hormones and the risk of osteoarthritis in women: epidemiological evidence
}

\author{
Michael C Nevitt, David T Felson
}

Current concepts of the pathogenesis of osteoarthritis suggest a role for both systemic predisposition and site specific mechanical factors. ${ }^{1}$ Sex hormones have long been considered a possible factor in the systemic predisposition to osteoarthritis, especially in women. ${ }^{2-5}$ There are several lines of epidemiological evidence suggesting that sex hormones, primarily oestrogen, play a role in osteoarthritis. These include a female excess in the prevalence and incidence of osteoarthritis that begins around the time of the menopause, and the association of prevalent osteoarthritis with possible markers of endogenous sex hormone exposure, including gynaecological surgery (reviewed elsewhere in this supplement) bone mass, and obesity. In a provocative review, Spector and Campion ${ }^{4}$ proposed that much of this evidence is consistent with the hypothesis that women with a greater exposure to endogenous oestrogen are predisposed to generalised osteoarthritis. On the other hand, studies that assess serum sex hormone levels in women with osteoarthritis are inconclusive. There is also a growing body of evidence to suggest that postmenopausal oestrogen replacement may protect against large joint osteoarthritis. It is possible that the effect of sex hormone exposure in osteoarthritis varies by menopausal status and stage in the extended pathogenesis of osteoarthritis.

\section{Menopause}

The prevalence of osteoarthritis in the hand, hip, and knee increases rapidly with age, beginning at about the age of 40-50 years in women, but less so in men, so that before the age of 50 , men have a higher prevalence of disease than women, but after 50 women have a higher prevalence, and the incidence and sex differences increase with age. ${ }^{6-10}$ Older women are also more likely to report joint symptoms for the same level of radiographic severity of knee osteoarthritis ${ }^{11}$ and have more rapid progression of hip osteoarthritis than men. ${ }^{12}$ Some investigators have suggested that women develop "menopausal arthritis" which consists of rapidly progressing osteoarthritis in the hand at the time of menopause. ${ }^{2313}$ Menopausal arthritis has been linked to generalised osteoarthritis with Heberden's nodes ${ }^{313}$ which may be more common in women. ${ }^{10}$ However, attempts to establish a temporal relationship between onset of generalised osteoarthritis and the menopause in individuals have been inconclusive. ${ }^{3}$ Nevertheless, these patterns are broadly consistent with a role for postmenopausal hormone deficiency in increasing the risk or severity of osteoarthritis in women. In diseases such as heart disease, gout, and osteoporosis in which, like osteoarthritis, women's risk of disease rises dramatically after the menopause, oestrogen loss has been strongly implicated in disease risk.

Menopausal changes in systemic hormone levels are complex and include a profound longterm decline in oestradiol concentrations, lesser declines in oestrone and androgen concentrations, increased ratios of oestrone and testosterone to oestradiol, and decreases in sex hormone binding globulin, as well as increased short term fluctuations in cyclical levels of several hormones during the perimenopausal period. ${ }^{14}{ }^{15}$ Hot flushes, the most common menopausal symptom, are strongly associated with musculoskeletal pain ${ }^{16}$ and appear to be linked to rapid fluctuations in serum oestradiol concentrations. ${ }^{15}$ Spector and Campion ${ }^{4}$ proposed that early perimenopausal declines in progesterone levels result in a temporary increase in levels of unopposed oestrogen which may predispose to osteoarthritis. Whether short term hormonal imbalances and fluctuations or permanent declines in hormone concentrations contribute to the surge in osteoarthritis risk which begins around the age of the menopause remains to be determined.

\section{Bone density and obesity}

The inverse relation of osteoarthritis with osteoporosis and the increased risk of osteoarthritis with obesity both suggest a possible role for

oestrogen in osteoarthritis pathogenesis. ${ }^{4}$ Oes-
Boston University, Massachusetts, USA

D T Felson 